\title{
What Have We Learned from SWAS?
}

\author{
Edwin A. Bergin ${ }^{1}$ and Gary J. Melnick ${ }^{2}$ \\ ${ }^{1}$ University of Michigan, Department of Astronomy, 825 Dennison, \\ 500 Church Street, Ann Arbor, MI 48109, USA \\ email: ebergin@umich.edu \\ ${ }^{2}$ Harvard-Smithsonian Center for Astrophysics, \\ 60 Garden Street, Cambridge, MA 02138, USA \\ email: gmelnick@cfa.harvard.edu
}

\begin{abstract}
The Submillimeter Wave Astronomy Satellite (SWAS) has recently completed 5.5 years of successful operation. Among the legacies of the mission has been a greater understanding of the abundance and spatial distribution of $\mathrm{H}_{2} \mathrm{O}$ and $\mathrm{O}_{2}$ within molecular clouds. We summarize SWAS results and discuss how the measured low abundance of water vapor and non-detections of molecular oxygen are suggestive of a general lack of atomic oxygen in the dense centers of molecular clouds. We also present a new more comprehensive model for the oxygen chemistry in the interstellar medium.
\end{abstract}

Keywords. astrobiology — astrochemistry — ISM: molecules — molecular processes — radiative transfer

\section{Introduction}

The Submillimeter Wave Astronomy Satellite (SWAS) was a mission primarily dedicated to the study of star formation, molecular cloud composition, and the structure of clouds. To carry out these investigations, $S W A S$ was designed to observe $\mathrm{H}_{2}^{16} \mathrm{O}, \mathrm{H}_{2}^{18} \mathrm{O}$, $\mathrm{O}_{2}, \mathrm{CI}$, and ${ }^{13} \mathrm{CO}$ in transitions that are either difficult or impossible to detect from within the Earth's atmosphere. SWAS is a complete radio telescope in space, capable of observing the transitions listed in Table 1 with a spectral resolution of $1 \mathrm{~km} \mathrm{~s}$ s$^{-1}$ (Melnick et al. 2000). At the time of launch, the primary mission goals were to answer three important questions:

(a) Where is all of the oxygen in the dense interstellar medium (and, in particular, are $\mathrm{H}_{2} \mathrm{O}$ and $\mathrm{O}_{2}$ major reservoirs of oxygen)?

(b) Are $\mathrm{H}_{2} \mathrm{O}$ and $\mathrm{O}_{2}$ significant gas coolants?

(c) What is the large-scale distribution of $\mathrm{C}$ I and warm ${ }^{13} \mathrm{CO}$ in molecular clouds?

During its 5.5-year mission, SWAS conducted pointed observations toward more than 300 distinct galactic sources and along more than 6800 lines of sight. In so doing, $S W A S$ data have been used to answer these questions. Page limitations prevent a comprehensive review of $S W A S$ 's contribution to each of these topics. Instead, this paper will focus on the greater understanding of $\mathrm{H}_{2} \mathrm{O}$ and $\mathrm{O}_{2}$ in quiescent gas within molecular cloud cores enabled by the $S W A S$ data.

\section{The Observations of $\mathrm{H}_{2} \mathrm{O}$ and $\mathrm{O}_{2}$ in Interstellar Clouds}

One of the primary scientific goals of $S W A S$ was to determine the abundance of water vapor in various components of the interstellar medium by observing the lowest rotational transitions of ortho- $\mathrm{H}_{2}^{16} \mathrm{O}$ and its isotopomer ortho- $\mathrm{H}_{2}^{18} \mathrm{O}$. The study of water vapor has several motivations. 
Grain Surface

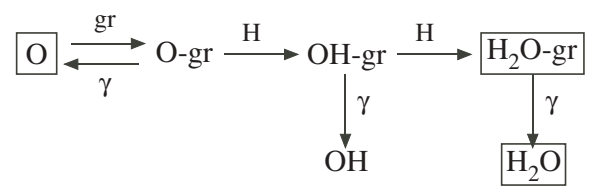

Gas Phase

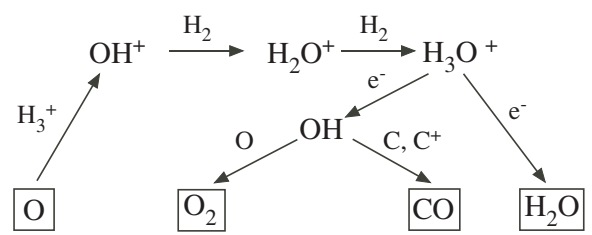

Figure 1. Key elements of the oxygen chemistry believed to occur on grain surfaces and in the cold gas phase. Photodesorption is denoted by $\gamma$. The boxed species are the most abundant and are of greatest interest in our study. O-gr, OH-gr, and $\mathrm{H}_{2} \mathrm{O}$-gr denote $\mathrm{O}, \mathrm{OH}, \mathrm{H}_{2} \mathrm{O}$, respectively, on grains.

Measurements of the water vapor abundance provide crucial constraints on astrochemical models that seek to elucidate the gas-phase chemical reactions and gas-grain interactions that determine the composition of the interstellar medium. In particular, chemical models (see Elitzur \& de Jong 1978; Neufeld et al. 1995) predict that for $T \gtrsim 300 \mathrm{~K}$, water vapor will account for most of the gas-phase oxygen that is not bound as CO, as a result of the neutral-neutral reactions: $\mathrm{H}_{2}+\mathrm{O} \rightarrow \mathrm{OH}+\mathrm{H}$ and $\mathrm{H}_{2}+\mathrm{OH} \rightarrow \mathrm{H}_{2} \mathrm{O}+\mathrm{H}$. At temperatures less than $300 \mathrm{~K}$, however, these reactions are negligibly slow because they possess significant activation energy barriers. Gas-phase water is then produced either by means of reactions on grain surfaces with subsequent sublimation or via cosmic-ray driven ion-neutral chemistry, as shown in Figure 1. Water formed on grains will remain frozen on the grain until either the grain temperature exceeds $\sim 100 \mathrm{~K}$ or the water molecule is photodesorbed by a UV photon. Gas-phase ion-neutral reactions (Herbst \& Klemperer 1973) proceed more slowly than the neutral-neutral reactions - requiring approximately $10^{6}$ years to reach chemical equilibrium in well-shielded gas with $n\left(\mathrm{H}_{2}\right) \sim 10^{5} \mathrm{~cm}^{-3}$ - and result in a maximum $\mathrm{H}_{2} \mathrm{O}$ abundance more than 100 times lower than in warmer gas.

\subsection{Water and Molecular Oxygen Abundances}

$S W A S$ has detected water vapor towards a diverse sample of astronomical objects. These objects include both dense and diffuse interstellar gas clouds, circumstellar envelopes, planetary atmospheres, and comets. As described below, the water abundances inferred from these $S W A S$ observations vary by more than a factor of $10^{5}$ and confirm some

Table 1. Spectral Lines Observed by $S W A S$

\begin{tabular}{cccccc}
\hline Species & Transition & $\begin{array}{c}\text { Energy Above } \\
\text { Ground State } \\
(E / k)\end{array}$ & $\begin{array}{c}\text { Frequency } \\
(\mathrm{GHz})\end{array}$ & $\begin{array}{c}\text { Wavelength } \\
(\mu \mathrm{m})\end{array}$ & $\begin{array}{r}\text { Critical } \\
\text { Density } \\
\left(\mathrm{cm}^{-3}\right)\end{array}$ \\
\hline $\mathrm{O}_{2}$ & $3,3-1,2$ & $26 \mathrm{~K}$ & 487.249 & 615.276 & $10^{3}$ \\
$\mathrm{C}_{\mathrm{I}}$ & ${ }^{3} \mathrm{P}_{1}-{ }^{3} \mathrm{P}_{0} \dagger$ & $24 \mathrm{~K}$ & 492.161 & 609.134 & $10^{4}$ \\
$\mathrm{H}^{18} \mathrm{O}$ & $1_{10}-1_{01} \dagger$ & $26 \mathrm{~K}$ & 547.676 & 547.390 & $10^{9}$ \\
${ }^{2} \mathrm{CO}$ & $J=5-4$ & $79 \mathrm{~K}$ & 550.926 & 544.161 & $2 \times 10^{5}$ \\
$\mathrm{H}_{2}{ }^{16} \mathrm{O}$ & $1_{10}-1_{01} \dagger$ & $27 \mathrm{~K}$ & 556.936 & 538.289 & $10^{9 \ddagger}$ \\
\hline
\end{tabular}

$\dagger$ Ground-state transition.

$\ddagger$ The critical density for $\mathrm{H}_{2}^{16} \mathrm{O}$ will likely be less than this value by a factor of $10^{2}-10^{4}$ due to significant radiation trapping in this line. The critical density for $\mathrm{H}_{2}^{18} \mathrm{O}$ could be reduced by a factor of $1.5-50$ due to the same effect. 


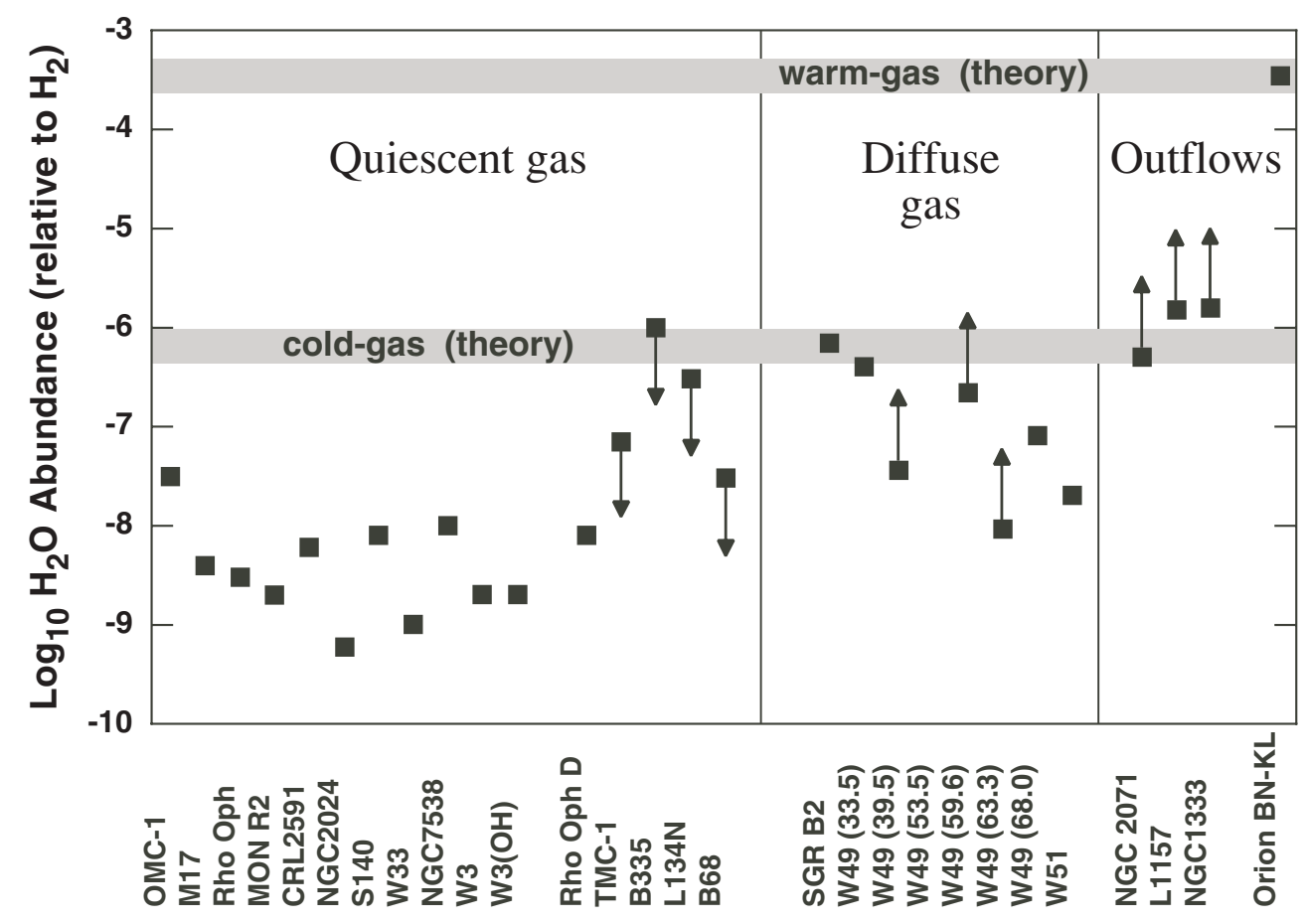

Figure 2. Measurements of the abundance of ortho-water vapor (relative to $\mathrm{H}_{2}$ ) towards molecular cloud cores labeled on the abscissa. Quiescent gas refers to abundances derived from water emission in dense ambient regions (taken from Melnick et al. 2000; Snell et al. 2000a,b,c). Diffuse gas refers to abundances derived from detections of o- $\mathrm{H}_{2} \mathrm{O}$ in absorption (Neufeld et al. 2000; Neufeld et al. 2002; Plume et al. 2004). Outflows abundances are from Melnick et al. (2000) and Neufeld et al. (2000).

aspects of previous theoretical predictions but also turn up some surprises. Figure 2 provides a graphical representation of the $S W A S$ water abundance determinations. In particular:

- In warm $(T \gtrsim 300 \mathrm{~K})$, dense $\left(n\left(\mathrm{H}_{2}\right) \gtrsim 10^{3} \mathrm{~cm}^{-3}\right)$ gas, the gaseous water abundance is $10^{-5}$ to $>10^{-4}$ relative to $\mathrm{H}_{2}$, in agreement with warm-cloud chemical models. Within this warm gas, $\mathrm{H}_{2} \mathrm{O}$ is a significant repository of oxygen as well as a major gas coolant, in accord with model predictions.

- In cold $(T \lesssim 30 \mathrm{~K})$, dense clouds the abundance of gaseous $\mathrm{H}_{2} \mathrm{O}$ is $\sim 100$ to 1000 times below the predictions of cold-cloud gas-phase chemical models and the $\mathrm{O}_{2}$ abundance is at least 100 times below predictions. As a result, gaseous $\mathrm{H}_{2} \mathrm{O}$ and $\mathrm{O}_{2}$ are neither significant reservoirs of oxygen in cold gas nor are they important gas coolants in such clouds.

- In cold diffuse $\left(n\left(\mathrm{H}_{2}\right)<10^{3} \mathrm{~cm}^{-3}\right)$ gas there is limited evidence that the $\mathrm{H}_{2} \mathrm{O}$ abundance is higher than in the cold dense gas, but $\mathrm{O}_{2}$ remains undetected.

\subsection{Water Radiation Transfer in the Effectively Thin Limit}

It is important to note that both $S W A S$ and Odin observed the ground state transition of ortho- $\mathrm{H}_{2} \mathrm{O}$. Water is a highly polar molecule and even small abundances can result in emission lines with high opacity. As such it is worth a discussion of the derivation of water vapor abundances under these conditions. Under normal circumstances large 
optical depth would make it highly difficult to extract reliable abundances. However, the emission from the $\mathrm{o}-\mathrm{H}_{2} \mathrm{O}$ ground state line is optically thick, but subthermal (given that densities are well below the critical density given in Table 1). Thus every photon that is created will scatter, but eventually escape the cloud (i.e. collisional de-excitations are rare). This so-called optically thick, but effectively thin limit is discussed by Linke et al. (1977) and Snell et al. (2000).

In the effectively thin limit the relation between the integrated emission and water vapor column density can be expressed analytically as:

$$
\int T_{R} d v=C n_{H_{2}} \frac{c^{3}}{2 \nu^{3} k} N\left(\mathrm{o}-\mathrm{H}_{2} \mathrm{O}\right) \frac{h \nu}{4 \pi} \exp \left(-h \nu / k T_{k}\right) .
$$

where $\mathrm{C}$ is the collisional de-excitation rate. With analysis of the source physical structure $\left(\mathrm{H}_{2}\right.$ volume density, total column density, and temperature) line-of-sight average abundances can be derived, which were presented in Figure 2 and summarized in $\S 2.1$.

Two additional methods can be used to constrain the water abundance: (1) Observations of the lesser abundant isotopologue, $\mathrm{H}_{2}^{18} \mathrm{O}$, can be used to reduce concerns regarding the opacity. This line has been detected by Odin in ambient gas in Orion by Olofsson et al. (2003) with resulting abundances that are in agreement with the earlier analysis using $\mathrm{H}_{2}^{16} \mathrm{O}$ (see also the discussion by Snell et al. 2000a); (2) One can use a-priori knowledge of the line-of-sight physical structure (density and temperature) and sophisticated Monte-Carlo radiation transfer models to constrain the water abundance. In this fashion average abundances for several well known objects were derived by Ashby et al. (2000), which again are in agreement with abundances derived assuming effectively thin emission. In addition, Bergin \& Snell (2002) linked chemistry to a radiation transfer model to derive sensitive abundance limits in two pre-stellar cores (B68 and $\rho$ Oph D) with well characterized density profiles. In these objects the average water abundance is $x\left(\mathrm{o}-\mathrm{H}_{2} \mathrm{O}\right)<3 \times 10^{-8}$.

\section{Gas-Grain Chemistry in the ISM}

In the initial interpretation of $S W A S$ results Bergin et al. (2000) examined the various factors that will influence the oxygen chemistry in quiescent gas. In the ISM it is very difficult to stop water production. In the gas phase all of the reactions on the sequence to make water and molecular oxygen have been measured in the lab (see Millar, this volume; Sims, this volume). Thus, if oxygen is present in the gas it will react with cosmic-rayproduced $\mathrm{H}_{3}^{+}$and quickly create both $\mathrm{H}_{2} \mathrm{O}$ and $\mathrm{O}_{2}$ with abundances much greater than the measured values of $\sim 10^{-9}$ and $<3 \times 10^{-7}$, respectively. Thus either water formation must be stunted, or our understanding of water destruction is incorrect.

Two potential solutions were isolated. Increased destruction could result if sufficient turbulent mixing were to expose water vapor to layers where photodissociation dominates or reactions with UV-produced $\mathrm{C}^{+}$and $\mathrm{C}$ are possible (Chièze \& Pineau Des Forêts 1989; Willacy, Langer, \& Allen 2002). While this solution may be relevant for some objects, it would not account for a lack of water vapor in objects dominated by thermal motions, such as B68 or other low-mass condensed cores (Bergin \& Snell 2002).

On the formation side, a solution examined by several groups to varying degrees (Bergin et al. 2000; Viti et al. 2001; Charnley, Rodgers, \& Ehrenfreund 2001; Roberts \& Herbst 2002 ) is to include the formation of water ice on grains (or depletion of oxygen from the gas). In this model, as presented by Bergin et al. in Figure 3, the fuel for the chemistry, oxygen atoms, is frozen onto grains in the form of water ice. This ice will not evaporate unless temperatures exceed 110-120 K (Fraser et al. 2001) and hence most oxygen is 


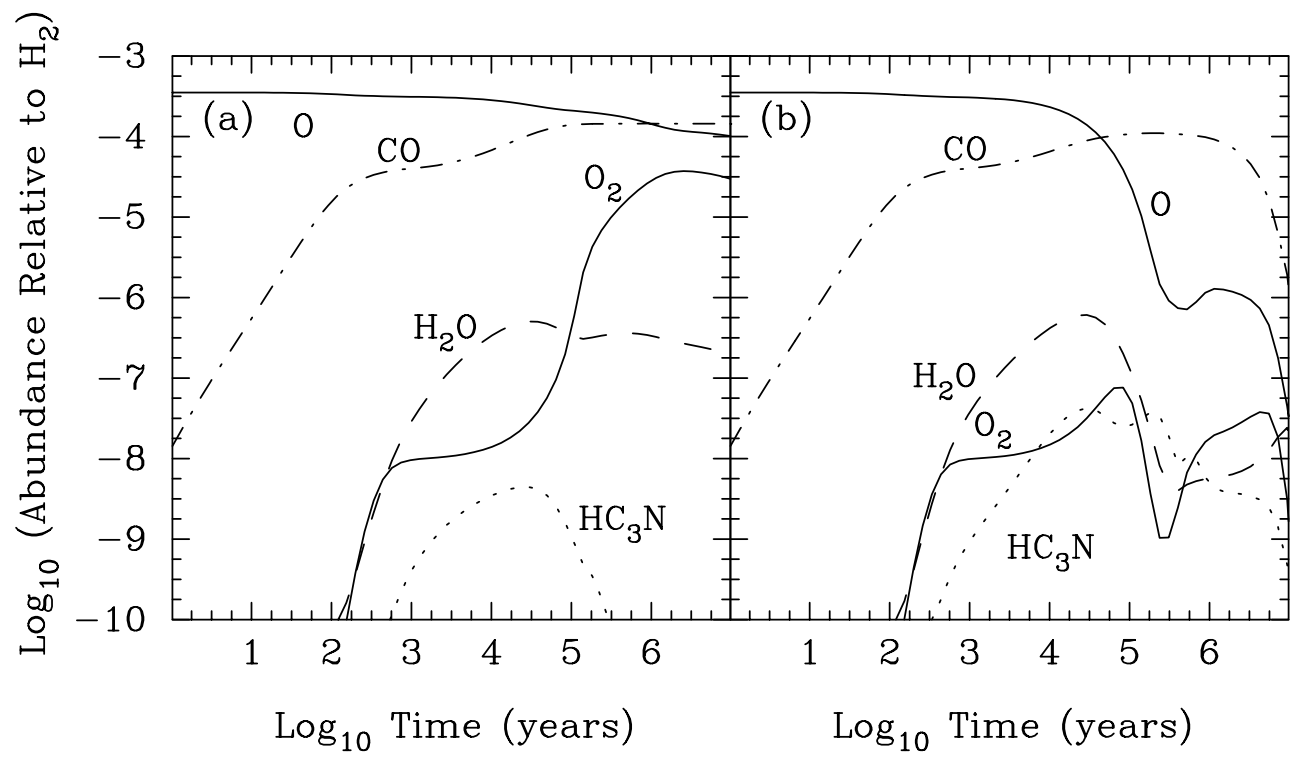

Figure 3. Abundances of $\mathrm{CO}, \mathrm{O}_{2}, \mathrm{H}_{2} \mathrm{O}$, and $\mathrm{HC}_{3} \mathrm{~N}$ as a function of time for two models of the gas-grain chemistry. (a) Model including depletion onto and desorption from grain surfaces, but no surface chemistry. (b) Model including depletion and desorption onto grains but with simple surface chemistry. Both models assume $\mathrm{n}_{H_{2}}=10^{5} \mathrm{~cm}^{-3}, \mathrm{~T}_{\text {gas }}=\mathrm{T}_{d u s t}=30 \mathrm{~K}$, and $\mathrm{A}_{V}=20^{m}$. The second model does not include any modifications to account for the discrete nature of grains, but is nonetheless illustrative of the effects of the process. Taken from Bergin et al. (2000).

unavailable to make water vapor or molecular oxygen in the gas. Thus, the low abundance of water vapor hints at a lack of gaseous atomic oxygen in the densest regions of molecular cloud cores. Even small amounts of oxygen in the gas would create water vapor that could be detected by $S W A S$ and Odin. In principle, this is in conflict with large atomic oxygen columns estimated from ISO [O I] observations towards many sources (Caux et al. 1999; Lis et al. 2001 and references therein). To account for the low abundances of water and molecular oxygen, this [O I] emission/absorption must be probing the outer, low-density, layers of the cloud. This is supported by an analysis of [O I] emission in $\rho$ Ophiuchi by Liseau et al. (1999), who find no enhancement of oxygen emission in dense cold clumps.

\section{A New Look at SWAS Observations}

With the perspective of more than 5 years of observations a number of factors become evident. First, despite a wide range of conditions probed (geometry, radiation field, temperature, density) water vapor observations of GMC cores showed, in general, line intensities of $T_{A}(557 \mathrm{GHz}) \sim 0.1-0.3 \mathrm{~K}$ (Orion A is one of the exceptions to this statement). This seems quite puzzling and hints at some general formation mechanism that is somehow insensitive to environment. Second, maps of emission over large spatial scales began to reveal compelling clues to the origin of water vapor emission. These maps readily demonstrate that water vapor is a surface tracer inside dense photodissociation regions (PDR's) and is not tracing the volume of the cloud.

An example of this is given in Figure 4 where we show $S W A S$ observations of NGC1333 presented in Bergin et al. (2003). The water emission in NGC1333 is divided into broad spectral lines that trace water entrained in molecular outflows (SS4, 6, 7, 8) and narrow 


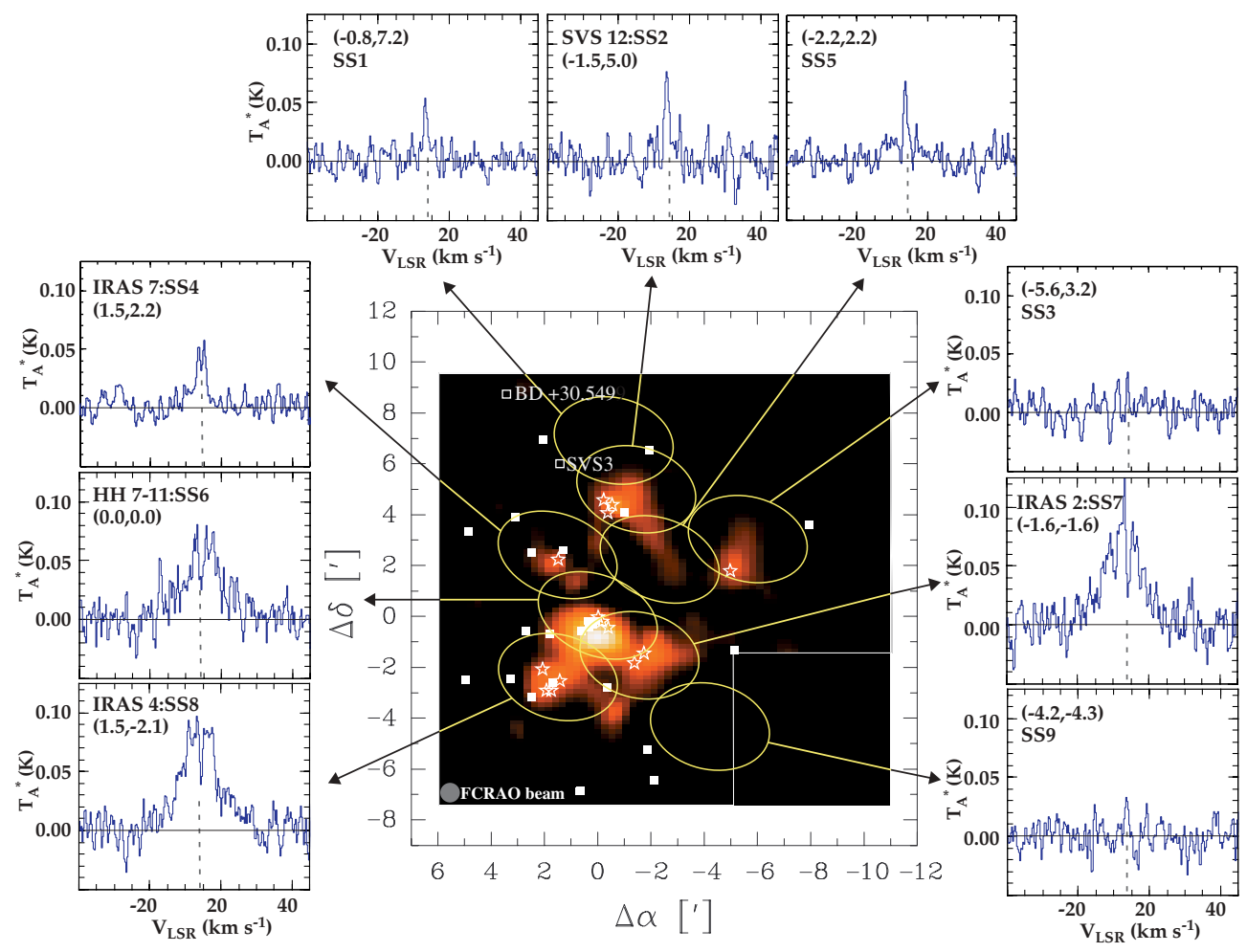

Figure 4. Spectra of the $1_{10}-1_{01} 556.936 \mathrm{GHz}$ transition of ortho-water vapor in NGC 1333 . Placements of the elliptical $S W A S$ beam are shown superposed on a map of integrated $\mathrm{N}_{2} \mathrm{H}^{+}$ $(\mathrm{J}=1-0)$ emission. The stars show the location of IRAS sources, while the solid squares are positions of known Herbig-Haro objects. Wide spectral lines are water emission arising from shocked gas, while narrow lines probe ambient material. Taken from Bergin et al. (2003).

$\left(\Delta v \sim 2-3 \mathrm{~km} \mathrm{~s}^{-1}\right)$ lines that probe ambient material (SS1, 2, 5). What is notable about the ambient water emission is that it does not appear to track the emission of $\mathrm{N}_{2} \mathrm{H}^{+}$(which is effectively a probe of the dense star-forming condensations). Rather the emission clusters to the north and is correlated with high- $J{ }^{13} \mathrm{CO}$ emission (see Bergin et al. 2003) that is directly associated with the luminous external heating sources (SVS3 and $\mathrm{BD}+30.549)$ which power the reflection nebula and the PDR. This was interpreted by Bergin et al. as undepleted water vapor present in low-density gas that gains emissivity due to heating in the PDR (see also Spaans \& van Dishoeck 2001). Extensive $S W A S$ observations of the Orion A molecular cloud also suggest water is present in the PDR. A comparison of $\mathrm{CN}, \mathrm{C}^{18} \mathrm{O}$, and water emission by Melnick et al. (in preparation) shows that water emission is highly correlated with CN, a known tracer of UV dominated gas in Orion (Rodríguez-Franco et al. 1998), and is uncorrelated with $\mathrm{C}^{18} \mathrm{O}$ emission, a tracer of total volume.

That water can be a tracer of regions with substantial UV radiation presents a puzzle because water is quite easily dissociated by UV photons (Yoshino et al. 1996). Hollenbach et al. (in preparation) present an appealing model, which asserts that toward cold clouds gaseous $\mathrm{H}_{2} \mathrm{O}$ exists only within a relatively narrow range of visual magnitudes near the cloud surface (see Figure 5). Closer to the surface than an $A_{\mathrm{V}}$ of a few, both $\mathrm{H}_{2} \mathrm{O}$ and $\mathrm{O}_{2}$ are photo-destroyed by the ambient galactic UV field. Deeper into the cloud than an $A_{\mathrm{V}}$ of $\sim 4-8\left(\Leftrightarrow N\left(\mathrm{H}+\mathrm{H}^{+}+\mathrm{H}_{2}\right) \simeq 0.8-1.6 \times 10^{22} \mathrm{~cm}^{-2}\right.$; c.f., Bohlin et al. 1978) $\mathrm{H}_{2} \mathrm{O}$, along 


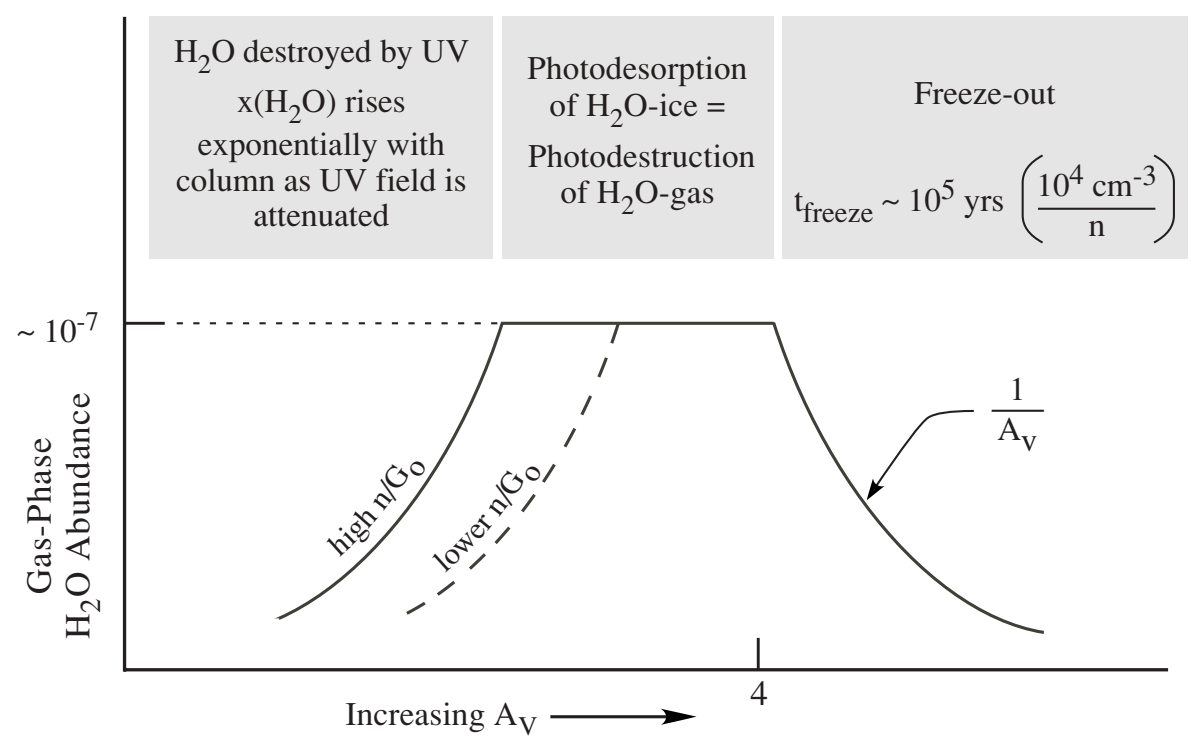

Figure 5. Schematic depiction of water vapor zone within molecular clouds.

with many other species, depletes onto grains. Between an $A_{\mathrm{V}}$ of 2 and 4-8 gas-phase abundances are set by the balance between photodesorption and photodestruction. The preferential depletion of several of the more abundant oxygen-bearing species serves to increase the gas-phase $\mathrm{C} / \mathrm{O}$ ratio deep within the cloud, further suppressing the gasphase production of $\mathrm{H}_{2} \mathrm{O}$ and $\mathrm{O}_{2}$ (e.g. Bergin et al. 2000). This picture is attractive since it naturally accounts for the low water vapor abundance inferred toward high column density lines of sight, the relatively higher water vapor abundances inferred toward lower column density lines of sight, and the high water-ice abundance derived toward many clouds.

By way of illustration, we consider a cloud of constant hydrogen density $n=10^{4} \mathrm{~cm}^{-3}$ illuminated by a somewhat enhanced ultraviolet radiation field $\mathrm{G}_{0}=10$, or about 10 times the average local interstellar field. The model results are shown in Figure 6 . As discussed above, the gas-phase oxygen is atomic at the surface because the ice is photodesorbed and O-bearing compounds in the gas phase are photodissociated to atomic form. However, the oxygen is mainly incorporated as water-ice deep in the cloud. The right panel in Figure 6 shows the threshold effect for $\mathrm{H}_{2} \mathrm{O}$ ice in the Taurus cloud. Plotted is the strength of the $\mathrm{H}_{2} \mathrm{O}$ ice absorption feature, measured using background sources that penetrate the cloud along a number of lines of sight, as a function of cloud column density or extinction. One sees a threshold column density ( $A_{V} \sim 4$ in Taurus) below which no ice feature is seen. Above this threshold, the absorption strength increases linearly with additional column density, and is consistent with all the elemental oxygen not in $\mathrm{CO}$ (or in refractory grain material like silicates) residing as water-ice (i.e., $x\left(\mathrm{H}_{2} \mathrm{O}\right.$-ice $) / x(\mathrm{H}$ nuclei) $\sim 10^{-4}$ ).

The gas-phase $\mathrm{O}_{2}$ and $\mathrm{H}_{2} \mathrm{O}$ abundances rise steeply from $A_{V}=0$ (the neutral cloud surface) to $A_{V}$ of a few as the photodissociation rates decline due to dust shielding from the UV field, and then the abundances decline as $A_{V}$ increases and all oxygen not in $\mathrm{CO}$ freezes out. In the deep interior, time-dependent chemistry must be considered since in steady state all the gas-phase oxygen, even that originally in $\mathrm{CO}$, is liberated and eventually incorporated into water-ice on grains. However, this process takes more than $3 \times 10^{6}$ years, or comparable to cloud lifetimes, so that CO generally is abundant 

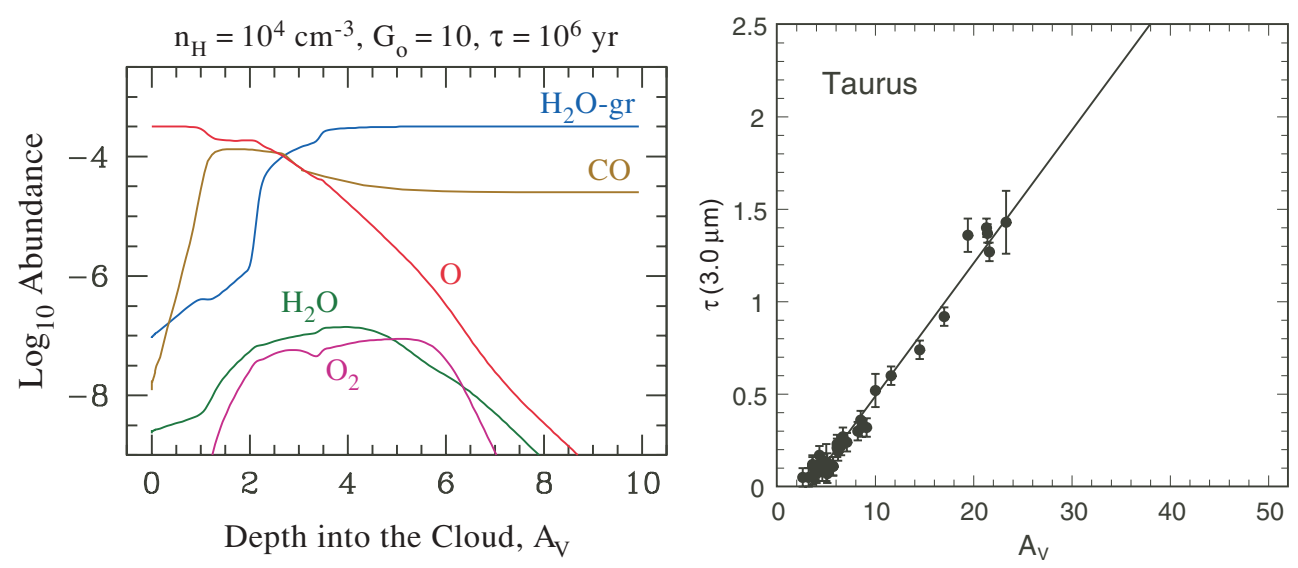

Figure 6. (Left) Chemical model predictions of the abundance of key oxygen-bearing species after $10^{6}$ years as a function of distance into a molecular cloud as measured in visual magnitudes of extinction, $A_{\mathrm{v}}$. (Right) The optical depth at $3 \mu \mathrm{m}$ due to $\mathrm{H}_{2} \mathrm{O}$-ice absorption versus the inferred depth into the Taurus (top) molecular cloud as measured in visual magnitudes of extinction, $A_{\mathrm{v}}$ (after Whittet et al. 2001).

in the gas phase as long as the grains are warmer than about $15-20 \mathrm{~K}$, the sublimation temperature for CO.

Thus, to understand the composition, this model requires that the depth dependence of the chemistry be treated. One must also include ice-forming grain surface chemistry, the change in grain surface area caused by ice formation, the ice desorption mechanisms especially photodesorption, and time-dependent chemistry. Hopefully, this picture will motivate further laboratory studies of photodesorption yields of various ice species as a function of UV wavelength.

\section{Conclusions}

Water vapor and molecular oxygen are key species in the interstellar medium because their abundances are sensitive to the chemistry that occurs in both the gas and solid state. During its 5.5-year mission, SWAS set clear limits on the cycle that creates water in interstellar space. We eagerly await Herschel observation of numerous transitions of both ortho- and para- $\mathrm{H}_{2} \mathrm{O}$, along with SOFIA observations of $\mathrm{H}_{2}^{18} \mathrm{O}$ that will further define the chemistry of oxygen, the third most abundant element in the Universe.

\section{Acknowledgements}

For the duration of the $S W A S$ mission this work was supported by NASA's $S W A S$ Grant NAS5-30702. We are grateful to all members of the $S W A S$ team who are too numerous to be named, but deserve thanks for supporting the proposal, design, development, software for the data pipeline and scheduling, data analysis tools, and the analysis summarized here.

\section{References}

Ashby, M.L.N., Bergin, E.A, Plume, R. et al. 2000, Ap. J. 539, L119

Bergin, E. A. \& Snell, R. L. 2002, Ap. J. 581, L105

Bergin, E. A., Kaufman, M. J., Melnick, G. J., Snell, R. L., \& Howe, J. E. 2003, Ap. J. 582, 830

Bergin, E.A., Melnick, G.J., Stauffer, J.R. et al. 2000, Ap. J. 539, L129 
Bohlin, R.C., Savage, B.D., \& Drake, J.F. 1978, Ap. J. 224, 132

Caux, E., et al. 1999, A\&A 347, L1

Charnley, S. B., Rodgers, S. D., \& Ehrenfreund, P. 2001, A\&3A 378, 1024

Chieze, J. P. \& Pineau Des Forets, G. 1989, A\&A 221, 89

Elitzur, M. \& de Jong, T. 1978, A\&A 67, 323

Fraser, H. J., Collings, M. P., McCoustra, M. R. S., \& Williams, D. A. 2001, M.N.R.A.S. 327, 1165

Herbst, E. \& Klemperer, W. 1973, Ap. J. 185, 505

Linke, R. A., Goldsmith, P. F., Wannier, P. G., Wilson, R. W., \& Penzias, A. A. 1977, Ap. J. 214,50

Lis, D. C., Keene, J., Phillips, T. G., Schilke, P., Werner, M. W., \& Zmuidzinas, J. 2001, Ap. J. 561,823

Liseau, R., et al. 1999, A\&A 344, 342

Melnick, G.J., Stauffer, J.R., Ashby, M.L.N. et al. 2000, Ap. J. 539, L77

Neufeld, D.A., Lepp, S., \& Melnick, G.J., 1995, Ap. J. Suppl. 100, 132

Neufeld, D.A., Ashby, M.L.N., Bergin, E.A. et al. 2000, Ap. J. 539, L111

Neufeld, D. A., Kaufman, M. J., Goldsmith, P. F., Hollenbach, D. J., \& Plume, R. 2002, Ap. J. 580,278

Olofsson, A. O. H., et al. 2003, Aध A 402, L47

Plume, R., et al. 2004, Ap. J. 605, 247

Roberts, H. \& Herbst, E. 2002, Astron. \& Ap., 395, 233

Rodriguez-Franco, A., Martin-Pintado, J., \& Fuente, A. 1998, A\&A A 329, 1097

Snell, R.L., Howe, J.E., Ashby, M.L.N. et al. 2000a, Ap. J. 539, L93

Snell, R.L., Howe, J.E., Ashby, M.L.N. et al. 2000b, Ap. J. 539, L97

Snell, R.L., Howe, J.E., Ashby, M.L.N. et al. 2000c, Ap. J. 539, L101

Spaans, M. \& van Dishoeck, E. F. 2001, Ap. J. 548, L217

Viti, S., Roueff, E., Hartquist, T. W., Pineau des Forêts, G., \& Williams, D. A. 2001, A\&̋A 370, 557

Whittet, D. C. B., Gerakines, P. A., Hough, J. H., \& Shenoy, S. S. 2001, Ap. J. 547, 872

Willacy, K., Langer, W. D., \& Allen, M. 2002, Ap. J. 573, L119

Yoshino, K., Esmond, J. R., Parkinson, W. H., Ito, K., \& Matsui, T. 1996, Chemical Physics 211,387

\section{Discussion}

Henning: Did you detect $\mathrm{H}_{2} \mathrm{O}$ in debris disks?

BERGin: We observed several debris disks (including $\beta$ Pic) for tens of hours and did not detect $\mathrm{H}_{2} \mathrm{O}$ or $[\mathrm{C} \mathrm{I}]$.

FrASER: Given that the $S W A S$ results indicate a lack of atomic O in the ISM gas, what are the implications of the low atomic abundance for subsequent complex chemistry especially on grains. I presume that hydrogenation of $\mathrm{O}$ will dominate, and a lot of $\mathrm{O}$ is locked up in $\mathrm{CO}+\mathrm{SiO}_{2}$ (in grains) - so how can we explain for e.g. $\mathrm{CO}_{2}$ ice observation and $\mathrm{CO}_{2}$ formation?

BERGin: My suspicion is that many of the complex species form with water ice in early stages when atomic hydrogen and oxygen are abundant. Once water formation stops at later denser stages, complex molecule production may occur more slowly.

Kroes: (1) What was the frequency of UV light in experiments showing water desorption? In Leiden, S. Andersson, I, and Ewine van Dishoeck see almost no water desorption in modeling of photodissociation of water in ice, in A-band, at $8.5 \mathrm{eV}$. (2) Why does $\mathrm{O}_{2}$ formation not accompany ice formation or if it does, why do you not observe $\mathrm{O}_{2}$ ? 
Bergin: (1) The experiment by Westley et al. (1995, Nature 373) was done using Ly $\alpha$ photons. (2) If water vapor and ice form predominantly at low extinction, the $\mathrm{O}_{2}$ is created but will be rapidly destroyed by UV photons.

GLASSGOLD: What is the role of $\mathrm{OH}$ observations in understanding the $S W A S$ and Odin results on $\mathrm{H}_{2} \mathrm{O}$ and $\mathrm{O}_{2}$ ?

BERGIN: $\mathrm{OH}$ has long been a suspected tracer of cloud surfaces and one model readily produces $\mathrm{OH}$ at the surface by UV photodissociation. It would be worthwhile to revisit this issue with direct $\mathrm{OH}$ observations in key sources such as Orion.

HerBst: What is the efficiency you need for $\mathrm{H}_{2} \mathrm{O}$ photo-desorption?

BERGIN: We have assumed a photo-desorption yield of 0.001 . If the water photo-desorption experiments in the lab are found to be much lower than the Westley et al. (1995, Nature 373 ) results then water can still be a surface tracer. In this case water would be present in lower-density envelopes surrounding the dense cores, in regions where oxygen has no time to collide with grains and react with surface $\mathrm{H}$ to form water ice.

Ceccarelli: I have a comment related to T. Henning's question about $S W A S$ nondetection of $\mathrm{H}_{2} \mathrm{O}$ in proto-planetary disks. We (Ceccarelli et al., Ap. J., in press) detected HDO in the disk of DM Tau. 